\title{
AFFINE PERMUTATIONS AND INVERSION MULTIGRAPHS
}

\author{
PAOLO PAPI \\ Dipartimento di Matematica, Istituto G. Castelnuovo, \\ Università degli studi di Roma "La Sapienza", \\ P.le Aldo Moro 5, 00185 Roma, Italy. \\ E-mail address: papi@mat.uniroma1.it
}

Submitted: October 4, 1996. Accepted: January 21, 1997.

\begin{abstract}
In this paper we solve the problem of characterizing inversion multigraphs (cf. $[\mathrm{BB}]$ ) associated to affine permutations.
\end{abstract}

\section{$\S 1$ InTRODUCTION AND BASIC DEFINITIONS}

Let $W$ be an affine Weyl group of type $\tilde{A}_{n-1} ; W$ is a group admitting the following presentation: generators $s_{1}, \ldots, s_{n}$ and defining relations:

$$
\begin{aligned}
s_{i}^{2} & =1 & & i=1, \ldots, n \\
s_{i} s_{i+1} s_{i} & =s_{i+1} s_{i} s_{i+1} & & i=1, \ldots, n-1 \\
s_{1} s_{n} s_{1} & =s_{n} s_{1} s_{n} . & &
\end{aligned}
$$

The elements of $W$ can be regarded as "affine permutations": in fact, as Lusztig observed in [L], the map $\Phi: W \rightarrow \tilde{S}_{n}$ defined on generators as $\Phi\left(s_{i}\right)=\pi_{i}, i=$ $1, \ldots, n$

$$
\pi_{i}(t)= \begin{cases}t & \bar{t} \neq \bar{i}, \overline{i+1} \\ t-1 & \bar{t}=\overline{i+1} \\ t+1 & \bar{t}=\bar{i}\end{cases}
$$

( $\bar{t}$ denotes the residue class of $t$ modulo $n$ ) is an isomorphism onto the following subgroup of permutations of the integers:

$$
\tilde{S}_{n}:=\left\{\pi: \mathbb{Z} \rightarrow \mathbb{Z} \mid \pi(t+n)=\pi(t)+n \forall t \in \mathbb{Z}, \quad \sum_{t=1}^{n} \pi(t)=\frac{n(n+1)}{2}\right\}
$$


It follows at once from the previous description that an element $\pi \in \tilde{S}_{n}$ is completely determined by the $\mathrm{n}$-tuple $[\pi(1), \ldots, \pi(n)]$.

In $[\mathrm{BB}]$ the authors develop a detailed analysis of the combinatorics of affine permutations; moreover they exhibit various encodings of affine permutations and minimal coset representatives of $\tilde{S}_{n} / S_{n}$. In connection with the weak Bruhat order, they describe affine permutations through certain inversion multigraphs and leave open the problem of classifying them.

In this paper we solve this last problem using our previous results on compatible orderings in root systems proceeding as follows. An inversion multigraph encodes an affine permutation $\pi$ by determining its "affine inversions"; from the point of view of root systems this amounts to describing the set of positive roots sent into negative ones by $\pi$. On the other hand, this kind of sets of positive roots has been combinatorially classified in $[\mathrm{P}]$ (where they are called compatible sets). So we translate the combinatorics of compatible sets into that of inversion multigraphs, working out some further simplifications; finally we obtain a characterization of the inversion multigraphs, involving weight conditions on certain triples of edges.

As a notational convention, we will denote by $\mathbb{N}$ (resp. $\mathbb{Z}_{+}$) the set of positive (resp. non-negative) integer numbers.

\section{$\S 2$ Affine Roots in the geometric Realization of $\tilde{S}_{n}$}

As any Coxeter group, $\tilde{S}_{n}$ can be faithfully represented as a transformation group (see e.g. [H], §5.3-5.4); for this consider the complex vector space $V$ with basis $\Pi=\left\{\alpha_{1}, \ldots, \alpha_{n}\right\}$ (the "simple roots") and let $W$ act on $V$ by linear extension of the following formula:

$$
s_{i}\left(\alpha_{j}\right)=\alpha_{j}-a_{i j} \alpha_{i}
$$

where the $a_{i j}$ 's are entries from the following $n \times n$ matrix:

$$
A=\left(a_{i j}\right)=\left(\begin{array}{cccccccc}
2 & -1 & 0 & 0 & \ldots & 0 & 0 & -1 \\
-1 & 2 & -1 & 0 & \ldots & 0 & 0 & 0 \\
0 & -1 & 2 & -1 & \ldots & 0 & 0 & 0 \\
\vdots & \vdots & \vdots & \vdots & \vdots & \vdots & \vdots & \vdots \\
0 & 0 & 0 & 0 & \ldots & -1 & 2 & -1 \\
-1 & 0 & 0 & 0 & \ldots & 0 & -1 & 2
\end{array}\right)
$$

Define the root system as the set of $W$-orbits in $V$ of the simple roots:

$$
\Delta:=W . \Pi
$$

Denote by $\Delta^{+}$the set of positive roots, i.e., the elements in $\Delta$ which are linear combinations with non-negative coefficients of the simple roots.

Note that $W$ acts on $V$ as a reflection group with respect to the bilinear form associated to the previous matrix (in the basis $\Pi$ ); this form is degenerate with 
1-dimensional kernel generated by the so-called fundamental imaginary root ${ }^{1}$ $\delta:=\sum_{i=1}^{n} \alpha_{i} . W$ permutes $\Delta$ and fixes $\delta$; moreover this linear action on $V$ is equivalent to an affine action on the subspace $V^{0}:=\oplus_{i=1}^{n-1} \mathbb{C} \alpha_{i}$; in this equivalence $W$ maps isomorphically to the semidirect product of $S_{n}$ with a group of "translations" (see $[\mathrm{K}]$, Prop. 6.5). Roots can be explicitly described as follows:

$$
\begin{aligned}
\Delta= \pm & \left(\left\{\alpha_{i}+\ldots+\alpha_{j}+k \delta \mid 1 \leq i \leq j \leq n-1, k \in \mathbb{Z}_{+}\right\} \cup\right. \\
& \left.\left\{-\left(\alpha_{i}+\ldots+\alpha_{j}\right)+h \delta \mid 1 \leq i \leq j \leq n-1, h \in \mathbb{N}\right\}\right) .
\end{aligned}
$$

For $\pi \in \tilde{S}_{n}$ introduce the set

$$
R_{\pi}:=\left\{\alpha \in \Delta^{+} \mid \pi(\alpha) \in \Delta^{-}\right\} .
$$

It is well known (see e.g. [H], §1.7) that if $s_{i_{1}} \ldots s_{i_{r}}$ is a reduced expression of $\pi \in \tilde{S}_{n}$, then

$$
R_{\pi}=\left\{\alpha_{i_{r}}, s_{i_{r}}\left(\alpha_{i_{r-1}}\right), s_{i_{r}} s_{i_{r-1}}\left(\alpha_{i_{r-2}}\right), \ldots, s_{i_{r}} \ldots s_{i_{2}}\left(\alpha_{i_{1}}\right)\right\} .
$$

Theorem 1. $([\mathrm{P}])^{2}$ A finite subset $L \subset \Delta^{+}$is of the form $R_{\pi}$ for some $\pi \in \tilde{S}_{n}$ if and only if it satisfies the following conditions:

(1) If $\lambda, \mu \in L$ and $\lambda+\mu \in \Delta$, then $\lambda+\mu \in L$.

(2) If $\lambda+\mu \in L, \lambda, \mu \in \Delta^{+}$, then $\lambda \in L$ or $\mu \in L$.

Moreover, $R_{\phi}=R_{\psi}$ if and only if $\phi=\psi$.

We will need in the following the explicit description of the action of $\pi \in \tilde{S}_{n}$ on $\Delta$ in the permutation realization. Suppose $\alpha \in \Delta^{+}$; then $\alpha$ is of the form $\alpha_{i}+\ldots+\alpha_{j}+k \delta$ or of the form $-\left(\alpha_{i}+\ldots+\alpha_{j}\right)+h \delta$ for some integers $1 \leq$ $i \leq j<n, k \geq 0, h \geq 1$. Let $q_{i}, r_{i}, i=1,2$ be the unique integers such that $\pi(i)=q_{1} n+r_{1}, \pi(j+1)=q_{2} n+r_{2},\left(q_{1}, q_{2} \in \mathbb{Z}, 0<r_{i} \leq n, i=1,2\right)$. Recall from [BB], $\S 3$, that $\pi(i) \equiv \pi(j) \bmod n \Leftrightarrow i \equiv j \bmod n$; so we can define

(3) $\pi\left(\alpha_{i}+\ldots+\alpha_{j}+k \delta\right)=\left(\alpha_{1}+\ldots+\alpha_{r_{2}-1}\right)-\left(\alpha_{1}+\ldots+\alpha_{r_{1}-1}\right)+\left(k-q_{1}+q_{2}\right) \delta$

(4) $\pi\left(-\left(\alpha_{i}+\ldots+\alpha_{j}\right)+h \delta\right)=\left(\alpha_{1}+\ldots+\alpha_{r_{1}-1}\right)-\left(\alpha_{1}+\ldots+\alpha_{r_{2}-1}\right)+\left(h+q_{1}-q_{2}\right) \delta$

By a straightforward verification, easily performed on generators using (1), (2), we get the following result.

Proposition 2. The previous formulas define an action of $\tilde{S}_{n}$ on $\Delta$; this action coincides with the reflection representation.

We finally recall the formula for the length function $\ell$ in $\tilde{S}_{n}$, affording the minimal number of Coxeter generators occurring in an expression of an affine permutation $\pi$; if square brackets denote the integer part of a rational number, we have (cf. [Shi], Lemma 4.2.2):

$$
\ell(\pi)=\sum_{1 \leq i<j \leq n}\left|\left[\frac{\pi(j)-\pi(i)}{n}\right]\right| .
$$

\footnotetext{
${ }^{1}$ This terminology is borrowed from the theory of Kac-Moody algebras [K]; note however that in our setting $\delta$ is not a root.

${ }^{2}$ In $[\mathrm{P}]$ the theorem is stated for subsets of positive roots in a finite crystallographic root system; however it obviously holds for finite subsets of roots in the affine case.
} 


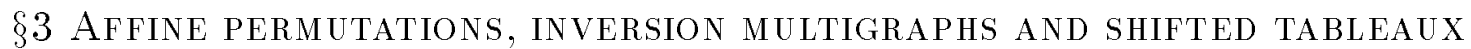

Let $\mathcal{G}_{n}$ denote the set of weighted oriented graphs having $\{1, \ldots, n\}$ as set of vertices; let then $A_{G}$ denote the set of oriented edges of an element $G \in \mathcal{G}_{n}$; if an edge $x \in A_{G}$ starts from the vertex $i$ and ends in the vertex $j$ we will denote it by $i \rightarrow j$; the corresponding weight will be denoted by $t(i, j)$ or $t(x)$.

An element $G \in \mathcal{G}_{n}$ is characterized by $A_{G}$ and the set of weights $\mathcal{W}_{G}=\{t(i, j) \mid$ $i \neq j$ \} (we enclose 0 among the weights, so $\mathcal{W}_{G}$ is a set consisting exactly of $n(n-1$ ) non-negative integer numbers).

Recall from $[\mathrm{BB}]$ the definition of the inversion multigraph $I_{\pi}$ relative to $\pi \in \tilde{S}_{n} . I_{\pi}$ is the element of $\mathcal{G}_{n}$ which has an edge of multiplicity $\left|\left[\frac{\pi(j)-\pi(i)}{n}\right]\right|$ between $i$ and $j$, directed towards the node with highest $\pi$-value, for all $1 \leq i<j \leq n$; edges of multiplicity zero are deleted. The figure below shows the inversion multigraph associated to $\pi=[4,5,-1,2] \in \tilde{S}_{4}$ :

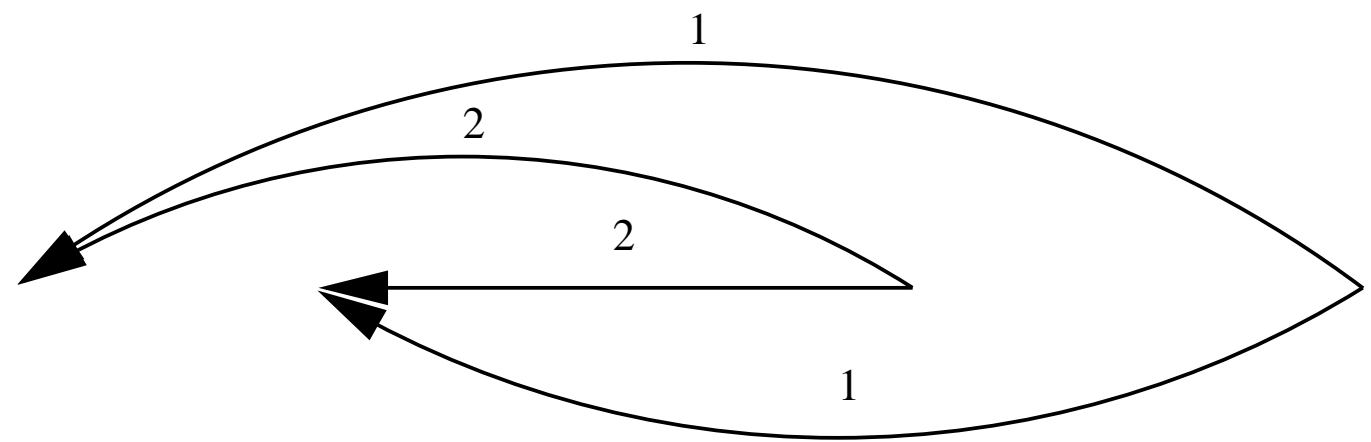

Note that , by formula $(5), \ell(\pi)=\sum_{x \in A_{I \pi}} t(x)$. Moreover, it is shown in [BB] that $I_{\pi}$ determines $\pi$; the problem of classifying inversion multigraphs is left open. In other words the map $\Lambda_{n}: \tilde{S}_{n} \rightarrow \mathcal{G}_{n}$ defined by $\Lambda_{n}(\pi)=I_{\pi}$ is injective and we have to find its image. By the very definitions it is clear that $\operatorname{Im}\left(\Lambda_{n}\right) \subseteq \mathcal{G}_{n}^{\prime}$ where

$$
\mathcal{G}_{n}^{\prime}:=\left\{G \in \mathcal{G}_{n} \mid \forall i, j \in\{1, \ldots, n\}, i \neq j, t(i, j) \neq 0 \Longrightarrow t(j, i)=0\right\} .
$$

From this remark it follows that we may organize the data appearing in an inversion multigraph in a slightly different way, which is more convenient for our goals. Let $\mathcal{T}_{n}$ denote the set of shifted tableaux of shape $\lambda=(n-1, n-2, \ldots, 1)$; we associate to a given element $\pi \in \tilde{S}_{n}$ a shifted tableau of shape $\lambda, T_{\pi}=$ $\left(\tau_{\pi}(i, j)\right)_{(i, j) \in I_{n}}, I_{n}:=\{(i, j) \mid 1 \leq i \leq j \leq n-1\}$, in the following way:

$$
\tau_{\pi}(i, j):=\left[\frac{\pi(j+1)-\pi(i)}{n}\right] .
$$

For instance, if $\pi=[4,5,-1,2] \in \tilde{S}_{4}$, then $T_{\pi}=\begin{array}{rrr}0 & -2 & -1 \\ & -2 & -1\end{array}$.

0

It is clear how to recover $I_{\pi}$ from $T_{\pi}$ and vice versa; we will prove that

$$
R_{\pi}=\left\{\alpha_{1}+\alpha_{2}, \alpha_{1}+\alpha_{2}+\delta, \alpha_{1}+\alpha_{2}+\alpha_{3}, \alpha_{2}, \alpha_{2}+\delta, \alpha_{2}+\alpha_{3}\right\}
$$


So, roughly speaking, the coefficient $\tau_{\pi}(i, j)$ in $T_{\pi}$ gives, according to its sign the number of roots of the string $\alpha_{i}+\ldots+\alpha_{j}+k \delta$ (or of the string $-\left(\alpha_{i}+\ldots+\alpha_{j}\right)+k \delta$ ) belonging to $R_{\pi}$. Recall that, using the algorithm described in [P], we may get a reduced expression of $\pi$ from the knowledge of $R_{\pi}$; in the example $\pi=s_{3} s_{4} s_{2} s_{1} s_{3} s_{2}$.

Set now, for $T \in \mathcal{T}_{n}$ :

$$
\begin{array}{r}
N_{T}:=\bigcup_{(i, j) \in I_{n}}\left(\left\{\alpha_{i}+\ldots+\alpha_{j}+k \delta \mid \tau(i, j)<0,0 \leq k<-\tau(i, j)\right\} \cup\right. \\
\left.\left\{-\left(\alpha_{i}+\ldots+\alpha_{j}\right)+h \delta \mid \tau(i, j)>0,1 \leq h \leq \tau(i, j)\right\}\right) .
\end{array}
$$

The following proposition, combined with the last statement of theorem 2, affords a proof of the injectivity of the map $\Lambda_{n}$; the proposition could be however deduced from the results of $[\mathrm{BB}], \S 3-4$.

Proposition 3. $R_{\pi}=N_{T_{\pi}}$.

Proof. For $(i, j) \in I_{n}$ introduce the sets

$$
\begin{aligned}
& J_{i j}^{+}:=\left\{\alpha_{i}+\ldots+\alpha_{j}+k \delta \mid k \geq 0\right\} \\
& J_{i j}^{-}:=\left\{-\left(\alpha_{i}+\ldots+\alpha_{j}\right)+h \delta \mid h \geq 1\right\} .
\end{aligned}
$$

We want to calculate the roots belonging to $\left(J_{i j}^{+} \cup J_{i j}^{-}\right) \cap R_{\pi}$. Suppose $\pi(i)=q_{1} n+r_{1}, \pi(j+1)=q_{2} n+r_{2}, q_{1}, q_{2} \in \mathbb{Z}, 0<r_{i} \leq n, i=1,2$ and $a \in J_{i j}^{+}, \tilde{a} \in J_{i j}^{-}$; then formulas (4), (5) imply the following relations:

$$
\begin{aligned}
& r_{1}>r_{2} \Longrightarrow\left\{\begin{array}{l}
\pi(a) \in \Delta^{-} \Leftrightarrow k \leq q_{1}-q_{2} \\
\pi(\tilde{a}) \in \Delta^{-} \Leftrightarrow h<q_{2}-q_{1}
\end{array}\right. \\
& r_{1}<r_{2} \Longrightarrow\left\{\begin{array}{l}
\pi(a) \in \Delta^{-} \Leftrightarrow k<q_{1}-q_{2} \\
\pi(\tilde{a}) \in \Delta^{-} \Leftrightarrow h \leq q_{2}-q_{1}
\end{array}\right.
\end{aligned}
$$

These relations clearly allow to determine $R_{\pi}$. In particular at most one of the two strings $J_{i j}^{+}, J_{i j}^{-}$has non-empty intersection with $R_{\pi}$; moreover:

$$
\operatorname{Card}\left(\left(J_{i j}^{+} \cup J_{i j}^{-}\right) \cap R_{\pi}\right)= \begin{cases}\left|q_{2}-q_{1}-1\right| & \text { if } r_{1}>r_{2} \\ \left|q_{2}-q_{1}\right| & \text { if } r_{1}<r_{2} .\end{cases}
$$

Now observe that

$$
\tau_{\pi}(i, j)=\left[\frac{\pi(j+1)-\pi(i)}{n}\right]=\left[\frac{\left(q_{2}-q_{1}\right) n+r_{2}-r_{1}}{n}\right]= \begin{cases}q_{2}-q_{1}-1 & \text { if } r_{1}>r_{2} \\ q_{2}-q_{1} & \text { if } r_{1}<r_{2}\end{cases}
$$

From this and the previous remarks we can easily deduce that $R_{\pi}=N_{T_{\pi}}$. 


\section{$\S 4$ THE MAIN RESULT}

We want to characterize the image of the map $\Lambda_{n}$; we have to translate conditions (1) and (2) of the theorem of section 2 into the combinatorics of inversion multigraphs. For $i, j \in\{1, \ldots, n\}, i \neq j$ set:

$$
\begin{gathered}
\mathcal{C}_{i \rightarrow j}:=\{(i \rightarrow k, k \rightarrow j) \mid k \neq i, j\} . \\
\tilde{t}(i, j):= \begin{cases}t(i, j) & \text { if } i<j \\
t(i, j)-1 & \text { if } i>j\end{cases}
\end{gathered}
$$

Now we can state our main theorem; we say for shortness that an (oriented) edge $i \rightarrow j$ is positive if $i<j$, negative otherwise.

Theorem 4. A multigraph $G \in \mathcal{G}_{n}^{\prime}$ belongs to $\operatorname{Im}\left(\Lambda_{n}\right)$ if and only if it satisfies the following conditions:

(I) $\forall i, j \in\{1, \ldots, n\}, i \neq j, \forall(x, y) \in \mathcal{C}_{i \rightarrow j} \cap\left(A_{G} \times A_{G}\right)$

$$
\tilde{t}(i, j) \geq \tilde{t}(x)+\tilde{t}(y) .
$$

(II) $\forall x \in A_{G}, \forall(a, b) \in \mathcal{C}_{x}, \forall(m, r): m+r=\tilde{t}(x)$

$$
\tilde{t}(a) \geq m \text { or } \tilde{t}(b) \geq r
$$

Here $m$ (resp. $r$ ) ranges over $\mathbb{N}$ or $\mathbb{Z}_{+}$according to whether a (resp. b) is positive or negative.

Remark. Condition (II) must be interpreted as follows. For each edge $x=i \rightarrow j$ in $G$, we have to consider all the paths starting from $i$ and ending in $j$ consisting of two edges, which do not necessarily belong to $A_{G}$ - in such a case we conventionally assign to them the weight $0-$; then the stated weight condition should be verified.

Proof. We want first to translate conditions (I), (II) on a multigraph $G \in \mathcal{G}_{n}^{\prime}$ into analogous conditions on the shifted tableaux $T=(\tau(i, j)) \in \mathcal{T}_{n}$ which encodes $G$ as previously explained.

Suppose for instance $(i, j) \in I_{n}, i \leq k<j$ and consider the relation

$$
(i \rightarrow k+1, k+1 \rightarrow j+1) \in\left(A_{G} \times A_{G}\right) \Longrightarrow \tilde{t}(i, j+1) \geq \tilde{t}(i, k+1)+\tilde{t}(k+1, j+1) .(*)
$$

Remark that the vertices $i, k+1$ and $k+1, j+1$ are joined by an edge if and only if $t(i, k+1) \neq 0, t(k+1, j+1) \neq 0$; considering the orientation of edges, the previous relations are equivalent to $\tau(i, k)>0, \tau(k+1, j)>0$. Therefore $(*)$ is equivalent to

$$
\tau(i, k)>0, \tau(k+1, j)>0 \Longrightarrow \tau(i, j) \geq \tau(i, k)+\tau(k+1, j)
$$


THE ElECTRonic JoURnal of COMBinatorics 4 (1997), \#R5

It is not hard to check that the following set of conditions is equivalent to (I), (II). (A) $\forall(i, j) \in I_{n}, \forall h, k, p: 1 \leq h<i \leq k<j<p \leq n-1$

$$
\begin{aligned}
\tau(i, k)>0, \tau(k+1, j)>0 & \Longrightarrow \tau(i, j) \geq \tau(i, k)+\tau(k+1, j) \\
\tau(i, k)<0, \tau(k+1, j)<0 & \Longrightarrow \tau(i, j) \leq \tau(i, k)+\tau(k+1, j)+1 \\
\tau(h, j)>0, \tau(h, i-1)<0 & \Longrightarrow \tau(i, j) \geq \tau(h, j)-\tau(h, i-1)-1 \\
\tau(h, j)<0, \tau(h, i-1)>0 & \Longrightarrow \tau(i, j) \leq \tau(h, j)-\tau(h, i-1) \\
\tau(i, p)>0, \tau(j+1, p)<0 & \Longrightarrow \tau(i, j) \geq \tau(i, p)-\tau(j+1, p)-1 \\
\tau(i, p)<0, \tau(j+1, p)>0 & \Longrightarrow \tau(i, j) \leq \tau(i, p)-\tau(j+1, p) .
\end{aligned}
$$

(B) If $\tau(i, j)<0$, set $s:=-\tau(i, j)-1$; then the following relations hold:

$\forall k: i \leq k<j, \forall(m, r) \in \mathbb{Z}_{+} \times \mathbb{Z}_{+}: m+r=s \quad \tau(i, k)<-m$ or $\tau(k+1, j)<-r$ $\forall h: 1 \leq h<i, \forall(m, r) \in \mathbb{Z}_{+} \times \mathbb{N}: m+r=s \quad \tau(h, j)<-m$ or $\tau(h, i-1) \geq r$ $\forall p: j<p \leq n-1, \forall(m, r) \in \mathbb{Z}_{+} \times \mathbb{N}: m+r=s \quad \tau(i, p)<-m$ or $\tau(j+1, p) \geq r$.

(B') If $\tau(i, j)>0$, set $s:=\tau(i, j)$; then the following relations hold:

$\forall k: i \leq k<j, \forall(m, r) \in \mathbb{N} \times \mathbb{N}: m+r=s \quad \tau(i, k) \geq m$ or $\tau(k+1, j) \geq r$ $\forall h: 1 \leq h<i, \forall(m, r) \in \mathbb{N} \times \mathbb{Z}_{+}: m+r=s \quad \tau(h, j) \geq m$ or $\tau(h, i-1)<-r$ $\forall p: j<p \leq n-1, \forall(m, r) \in \mathbb{N} \times \mathbb{Z}_{+}: m+r=s \quad \tau(i, p) \geq m$ or $\tau(j+1, p)<-r$.

On the other hand conditions (A) and (B), (B') correspond to relations (1) and (2) of the theorem of section 2 respectively. Let us explain how to recover the former set of relations from the latter.

Remark that, given an affine root $\left(\alpha_{i}+\ldots+\alpha_{j}\right)+s \delta \in \Delta^{+}, s \in \mathbb{Z}_{+}$, its decompositions into the sum of two positive roots are only the following ones:

$$
\begin{array}{ll}
\left(\alpha_{i}+\ldots+\alpha_{k}+m \delta\right)+\left(\alpha_{k+1}+\ldots+\alpha_{j}+r \delta\right), & (m, r) \in \mathbb{Z}_{+} \times \mathbb{Z}_{+}: m+r=s \\
\left(\alpha_{h}+\ldots+\alpha_{j}+m \delta\right)+\left(-\left(\alpha_{h}+\ldots+\alpha_{i-1}\right)+r \delta\right),(m, r) \in \mathbb{Z}_{+} \times \mathbb{N}: m+r=s \\
\left(\alpha_{i}+\ldots+\alpha_{p}+m \delta\right)+\left(-\left(\alpha_{j+1}+\ldots+\alpha_{p}\right)+r \delta\right),(m, r) \in \mathbb{Z}_{+} \times \mathbb{N}: m+r=s
\end{array}
$$

where $i \leq k<j, 1 \leq h<i, j<p \leq n-1$. The analogous relations for a root of the form $-\left(\alpha_{i}+\ldots+\alpha_{j}\right)+s \delta \in \Delta^{+}, s \in \mathbb{N}$ are the following:

$$
\begin{array}{ll}
\left(-\left(\alpha_{i}+\ldots+\alpha_{k}\right)+m \delta\right)+\left(-\left(\alpha_{k+1}+\ldots+\alpha_{j}\right)+r \delta\right), & (m, r) \in \mathbb{N} \times \mathbb{N}: m+r=s \\
\left(-\left(\alpha_{h}+\ldots+\alpha_{j}\right)+m \delta\right)+\left(\alpha_{h}+\ldots+\alpha_{i-1}+r \delta\right), & (m, r) \in \mathbb{N} \times \mathbb{Z}_{+}: m+r=s \\
\left(-\left(\alpha_{i}+\ldots+\alpha_{p}\right)+m \delta\right)+\left(\alpha_{j+1}+\ldots+\alpha_{p}+r \delta\right), & (m, r) \in \mathbb{N} \times \mathbb{Z}_{+}: m+r=s
\end{array}
$$

$(i \leq k<j, 1 \leq h<i, j<p \leq n-1)$. 
Note further that the sum of two positive roots is again a root if and only if we are in one of the previous cases (for some $(i, j) \in I_{n}$ ).

Now assume $T=T_{\pi}$ for $\pi \in \tilde{S}_{n}$. If, for instance, $\tau(i, k)>0, \tau(k+1, j)>0$ then, by proposition 3 , the roots $-\left(\alpha_{i}+\ldots+\alpha_{k}\right)+\tau(i, k) \delta,-\left(\alpha_{k+1}+\ldots+\alpha_{j}\right)+\tau(k+1, j) \delta$ belong to $R_{\pi}$. So (1) forces also $\left(-\left(\alpha_{i}+\ldots+\alpha_{j}\right)+(\tau(i, k)+\tau(k+1, j)) \delta\right)$ to belong to $R_{\pi}$. Therefore, again by proposition 3 , the relation $\tau(i, j) \geq \tau(i, k)+\tau(k+1, j)$ must hold. Small variations of this argument imply the other relations in (A).

Condition (2) means that, if a root $x=-\left(\alpha_{i}+\ldots+\alpha_{j}\right)+k \delta$ (resp. $y=$ $\alpha_{i}+\ldots+\alpha_{j}+k \delta$ ) belongs to $R_{\pi}$, then for each decomposition of $x$ (resp. $y$ ) into the sum of two positive roots at least one component belongs to $R_{\pi}$; it is easy to prove that it suffices to check this property when $k$ is the greatest integer such that a root of the form $-\left(\alpha_{i}+\ldots+\alpha_{j}\right)+r \delta$ or $\alpha_{i}+\ldots+\alpha_{j}+r \delta$ belongs to $R_{\pi}$ : so if $\tau(i, j)<0$ (resp. $\tau(i, j)>0$ ) then conditions (B) (resp. (B')) must hold. We have finally proved that inversion multigraphs satisfy the conditions in the statement of the theorem; on the other hand we claim that, if a multigraph $G$ verifies (I) and (II) and $T$ is the associated shifted tableau, then $N_{T}$ is a compatible set. Let us verify condition (1) of theorem 2; suppose that $\alpha_{i}+\ldots+\alpha_{k}+r \delta, \alpha_{k+1}+\ldots+\alpha_{j}+s \delta$ $1 \leq i \leq k<j \leq n-1, r, s \in \mathbb{Z}_{+}$belong to $N_{T}$ : we have to prove that $\alpha_{i}+\ldots+$ $\alpha_{j}+(r+s) \delta \in N_{T}$. We know that $r<-\tau(i, k), s<-\tau(k+1, j)$ : then condition (A) (which is equivalent to (I)) implies

$$
\tau(i, j) \leq \tau(i, k)+\tau(k+1, j)+1<-r-s
$$

and in turn we get $\alpha_{i}+\ldots+\alpha_{j}+(r+s) \delta \in N_{T}$. The other cases relative to condition (1) and condition (2) can be verified in a similar way.

Since $N_{T}$ is compatible, there exists an (unique) element $\pi \in \tilde{S}_{n}$ such that $N_{T}=R_{\pi}$. Therefore $\Lambda_{n}(\pi)=G$ and the proof is completed.

Examples. Consider the following multigraphs:
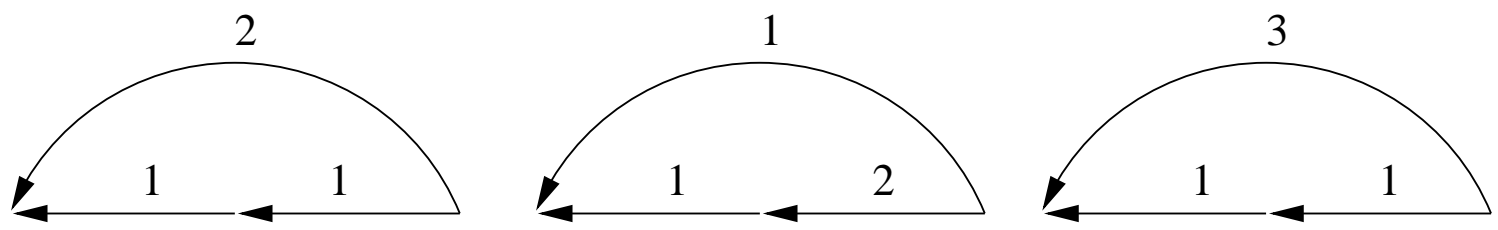

The first satisfies the conditions of theorem 4: in fact it encodes the affine permutation $\pi=[4,2,0]$. The other two graphs are not associated to affine permutations since they satisfy exactly one of the conditions in the previous theorem (the second does not satisfy (I) whereas the third does not satisfy (II)). 


\section{REFERENCES}

[BB] A. Björner, F. Brenti, Affine permutations of type A, The electronic journal of combinatorics 3(2) (1995).

[H] J.E. Humphreys, Reflection groups and Coxeter groups, Cambridge University Press, Cambridge, 1990.

[K] V.G. Kac, Infinite Dimensional Lie Algebras, Birkhäuser, Boston, 1983.

[L] G. Lusztig, Some examples of square integrable representations of semisimple p-adic groups, Trans. Amer. Math. Soc. 277 (1983), 99-111.

[P] P. Papi, A characterization of a special ordering in a root system, Proc. Amer. Math. Soc. 120 (1994), 661-665.

[Shi] Shi Jian-yi, The Kazdhan-Lusztig cells in certain affine Weyl groups, Springer, Berlin, 1986, Lect. Notes in Math. 1179. 